\title{
Mechanisms of cadmium-induced chronotoxicity in mice
}

\author{
Nobuhiko Miura ${ }^{1}$, Atsushige Ashimori1,2, Asuka Takeuchi 1,2, Katsumi Ohtani, \\ Naoko Takada², Yukie Yanagiba', Masaharu Mita ${ }^{4}$, Masako Togawa ${ }^{5}$ and Tatsuya Hasegawa ${ }^{5}$ \\ ${ }^{1}$ Division of Health Effects Research, Japan National Institute of Occupational Safety and Health, \\ 6-21-1, Nagao, Tama-ku, Kawasaki 214-8585, Japan \\ ${ }^{2}$ Laboratory of Environmental Soil Science, School of Life and Environmental Science, Azabu University, 1-17-71, \\ Fuchinobe, Chuo-ku, Sagamihara 252-5201 Japan \\ ${ }^{3}$ Division of Hazard Evaluation and Epidemiology Research, Japan National Institute of Occupational Safety and Health, \\ 6-21-1, Nagao, Tama-ku, Kawasaki 214-8585, Japan \\ ${ }^{4}$ Laboratory Animal Research Center, School of Pharmaceutical Sciences, Kitasato University, \\ 5-9-1, Shirokane, Minato-ku, Tokyo, 108-8642, Japan \\ ${ }^{5}$ Department of Environmental Biochemistry, Yamanashi Institute of Environmental Sciences, 5597-1, \\ Kenmarubi, Kamiyoshida, Fujiyoshida, Yamanashi 403-0005, Japan
}

(Received October 10, 2013; Accepted October 23, 2013)

\begin{abstract}
Biological defense factors show diurnal variations in their expression levels or activities. These variations can induce the different sensitivity to external toxicants of a day. We reported earlier that mice showed clear diurnal variation of cadmium (Cd)-induced toxicity, i.e., chronotoxicity. In this report, we investigated additional new evidences for the cadmium $(\mathrm{Cd})$-induced chronotoxicity, and considered the mechanisms contributed to this chronotoxicity. Male C57BL/6J mice were injected with $\mathrm{CdCl}_{2}$ $(6.4 \mathrm{mg} / \mathrm{kg}$, one shot) intraperitoneally at 6 different time points of a day (zeitgeber time (ZT); ZT2, ZT6, ZT10, ZT14, ZT18 or ZT22) followed by monitoring the mortality until 14 days after the injection. We observed extreme difference in survival numbers: surprisingly, all mice died at ZT2 injection while all mice survived at ZT18 injection. Moreover, in non-lethal dose of Cd $(4.5 \mathrm{mg} / \mathrm{kg})$, the values of alanine aminotransferase (ALT) and aspartate aminotransferase (AST) used as indexes of hepatotoxicity markedly increased at ZT6 injection while mostly unchanged at ZT18 injection. To consider the mechanisms of this extreme diurnal variation, we examined biochemical studies and concluded that the diurnal variation was not caused by the differences in hepatic Cd level, basal hepatic metallothionein (MT) level, and induction level or induction speed of hepatic MT. We suggested that one of the candidate determination factors was glutathione. We believe that the "chronotoxicology" for metal toxicity may be classic, yet new viewpoint in modern toxicology field.
\end{abstract}

Key words: Chronotoxicology, Diurnal variation, Biological rhythm, Cadmium, Glutathion, Metallothionein

\section{INTRODUCTION}

It is well known that biological defense factors show diurnal variation in their expression levels or their activities. For example, biological responses to various drugs used for antitumor, antiangiogenic, antiasthma or antihypertensive show 24-hr rhythmicity (Peters et al., 1987; Koyanagi et al., 2003; Hermida et al., 2005; Burioka et al., 2010). This diurnal variation may depend on alterations in drug metabolism activities, e.g. bioactivation or bioinactivation by cytochrome P450 enzymes (Froy,
2009). Furthermore, expressions of many drug processing genes show diurnal variation (Zhang et al., 2009). These facts investigate that administration timing varies the drug efficacy, i.e., effectiveness (medicinal benefit) and toxicity (adverse effect). This viewpoint, the diurnal variation of drug sensitivity, has been applied for many years into the medicinal field as chronopharmacology and chronotherapy (Burioka et al., 2010; Flack and Nasser, 2011).

In the toxicological field, however, insufficient attention about this viewpoint has been paid, especially heavy metal compounds. We believe that this viewpoint, the

Correspondence: Nobuhiko Miura (E-mail: miuran@h.jniosh.go.jp) 
diurnal variation of metal sensitivity (toxicity), should be introduced into toxicology as "chronotoxicology" becoming indispensable in the future toxicology. We predict that toxic severity caused by heavy metal exposure is different when quantitative changes of the biological defense factors occurs; e.g., toxicity will arise strongly when biological defense functions are low (for their levels and/or activities). Therefore it is possible that the severity of toxicity would differ by exposure timing. Actually, the diurnal variation of cadmium $(\mathrm{Cd})$-induced mortality was recently reported by our study in which ICR mice were sensitive to $\mathrm{Cd}$ acute toxicity during light phase, while tolerant during dark-phase (Miura et al., 2012). We also reported that $\mathrm{Cd}$-induced testicular toxicity showed diurnal variation (Ohtani et al., 2013). Cd has been used in various industrial products such as electrodes, pigments, coatings and alloys/control rods for nuclear reactors, and is classified as a human carcinogen (Wade et al., 1993; Pinot et al., 2000; Miura, 2009). Although usage amounts and applications of $\mathrm{Cd}$ have been considerably decreasing by the laws and regulations such as RoHS, Cd has been utilized in various industrial products even today and has also been produced as a by-product in refining processes of zinc. Moreover, because "shift work with night work" has adopted and has been an indispensable working arrangement especially into manufacturing industries, shift workers work at day time and night time. This means that shift workers have possibilities to be exposed to toxic substances at various times of a day. Therefore, we should pay attention to the $\mathrm{Cd}$-induced chronotoxicity, because shift workers have occasions to expose at various times of a day, additionally, have a risk for exposure to high concentration of $\mathrm{Cd}$ by accidental exposure.

In this report, in order to demonstrate additional evidences for the diurnal variation of $\mathrm{Cd}$-induced toxicity, we used another strain of mice $(\mathrm{C} 57 \mathrm{BL} / 6 \mathrm{~J})$ and investigated the diurnal variation of hepatotoxicity by non-lethal dose of $\mathrm{Cd}$. Furthermore, we considered the mechanisms of this chronotoxicity from the results of biochemical studies.

\section{MATERIAL AND METHODS}

\section{Animal studies}

Male C57BL/6J mice (5 weeks of age, 23-25 g body weight) were purchased from Nihon Clea (Clea Japan, Inc., Tokyo, Japan), and were kept under standard conditions with controlled temperature $\left(24 \pm 1^{\circ} \mathrm{C}\right)$, humidity $(55 \pm 5 \%)$ and light $(12: 12 \mathrm{hr}$ light/dark cycles, lights on at 08:00 AM; LD room). The lights were white fluorescent lamp, about 100-150 lux intensity at the lev- el of cages. In order to make possible the $\mathrm{Cd}$ injection at night-time during daytime (our working hours), we prepared the "DL room" in which light/dark cycles were reversed (lights on at 08:30 PM; lights off at 08:30 AM). Mice were adapted for 14 days with assigned to 6 groups of 5 animals in an appropriate room (3 cages were in LD room, 3 cages were in DL room). All animals had free access to sterilized commercial pellet diet (CE-2, Clea Japan Inc., Tokyo, Japan) and filter-sterilized tap water.

To determine the injection-time dependent difference of mortality, male C57BL/6J mice ( 7 weeks of age, $\mathrm{n}=5$ ) were received intraperitoneally (i.p.) with single injection of $6.4 \mathrm{mg} / \mathrm{kg}$ of $\mathrm{CdCl}_{2}(0.1 \mathrm{ml} / 10 \mathrm{~g}$ body weight; Wako chemical, Tokyo, Japan) at 6 different time points (zeitgeber time (ZT); ZT2, ZT6, ZT10, ZT14, ZT18 or ZT22). $Z T$ shows elapsed time from the lighting on. In the case of dark period (ZT14, ZT18 and ZT22), injections were performed in the DL room under red light. The intensity of red light was less than 5 lux at the level of cages, and was approximately 10 lux at the time of injection by bringing animals close to the light (about $2 \mathrm{sec}$ ). The mortalities were monitored until 14 days after the injection. All symptoms and deaths were observed every day during 14 days monitoring. After experiment, all mice were sacrificed using carbon dioxide.

In time course experiments, male $\mathrm{C} 57 \mathrm{BL} / 6 \mathrm{~J}$ mice ( 7 weeks of age, $n=5$ ) were received single injection of $\mathrm{CdCl}_{2}$ (4.5 mg/kg; i.p.), and were dissected at 2, 6, 12, and $24 \mathrm{hr}$ after the injection with being anesthetized using ether. Control mice (for $0 \mathrm{hr}$ ) were received same volume ( $0.1 \mathrm{ml} / 10 \mathrm{~g}$ body weight) of saline. Liver samples were collected and stored at $-80^{\circ} \mathrm{C}$ until analyses of $\mathrm{Cd}$ concentrations, $\mathrm{Cd}$ distributions, and levels of several biological factors. Blood samples were also collected from inferior vena cava using Supercath (Medikit Co. ltd., Tokyo, Japan) which needle was pre-saturated with heparin, and were centrifuged at $1,000 \times \mathrm{g}\left(4^{\circ} \mathrm{C}, 10 \mathrm{~min}\right)$ to separate plasma samples. Plasma samples were stored at $-80^{\circ} \mathrm{C}$ until analyses of levels of alanine aminotransferase (ALT) and aspartate aminotransferase (AST) as indexes of hepatotoxicity.

For estimation of diurnal variation of basal glutathione (GSH) and metallothionein (MT) levels in the liver, male C57BL/6Jmice ( 7 weeks of age, $n=5$ ) which had not been received any administrations were dissected at 6 different ZT (2, 6, 10, 14, 18 or 22). Liver samples were collected followed by determining the GSH and MT protein levels (described below).

\section{GSH and MT levels}

For determination of the hepatic GSH levels, liver sam- 
Cadmium chronotoxicity and glutathione

ples were homogenized in 5\% sulfosalicylic acid (SSA) for $30 \mathrm{sec}$ in ice-water bath. The homogenates consisting of $100 \mathrm{mg}$ liver in $1 \mathrm{ml}(10 \%)$ were centrifuged at 8,000 $\mathrm{x}$ g for $10 \mathrm{~min}$ at $4^{\circ} \mathrm{C}$ to remove proteins. Supernatants were assayed for reduced-form of GSH using GSSG/GSH Quantification Kit (Dojindo Laboratories, Kumamoto, Japan) according to the manufacture's instruction.

The hepatic MT protein levels were determined by $\mathrm{Hg}-$ binding assay (Kobayashi et al., 2007). Mercury bound to MT was measured using a mercury analyzer RA-2A (Nippon Instruments, Tokyo, Japan). The MT content was expressed as nanomoles of mercury bound.

\section{GSH depletion}

Male C57BL/6J mice were given free access to drinking water supplemented by $20 \mathrm{mM}$ buthionine sulfoximine (BSO; Sigma-Aldrich Japan, Tokyo, Japan) for 4 days to prevent GSH synthesis continuously (Watanabe et al., 2003), with modification based on the information from the subject and personal communication at toxicological conference held in Japan at 2011. Liver samples were collected 4 days after the BSO treatment (at ZT6 and ZT18) followed by determination of GSH contents. Another groups of mice which administered the BSO or non-treated water (its control) were injected with $\mathrm{CdCl}_{2}$ (4.5 mg/kg; i.p.), and were sacrificed $24 \mathrm{hr}$ after the injection. Collection and storage of the blood samples were performed as described above.

\section{Measurement of ALT and AST activities}

Activities of ALT and AST in plasma were measured using Transaminase CII Test Wako (Wako chemical, Tokyo, Japan) according to the manufacture's instruction. Briefly, using 96 multiwell plate, the plasma samples $(1 \mu 1)$ were mixed with $50 \mu 1$ of substrate mixture (an equal volume of each Substrate buffer and Coloring reagent), incubated at $37^{\circ} \mathrm{C}$ for 20 min followed by addition of Stopping solution $(100 \mu \mathrm{l})$. The absorbance of blue pigment formed by series of this reaction was measured at $540 \mathrm{~nm}$. For relative quantification, calibration curve was prepared using ALT or AST Standard solution by serial dilution.

\section{Accumulation and distribution of $\mathrm{Cd}$ in the liver after $\mathrm{CdCl}_{2}$ treatment}

Liver samples were digested with nitric acid. The concentration of $\mathrm{Cd}$ in each sample was determined using an inductively coupled plasma mass spectrometry (ICPMS; HP4500, Yokokawa Analytical Systems, Japan). Cd concentration was determined at the mass numbers of $111 \mathrm{~m} / \mathrm{z}$.
The distribution profiles of $\mathrm{Cd}$ in the soluble fraction of the liver were analyzed using high performance liquid chromatography-ICP-MS (HPLC/ICP-MS) (Miura et al., 2006). Liver samples which obtained from 2, 6, 12, and $24 \mathrm{hr}$ after the single injection of $\mathrm{CdCl}_{2}$ as described above were homogenized in five volumes of saline followed by ultracentrifugation at $105,000 \times \mathrm{g}$ for $1 \mathrm{hr}$ at $4{ }^{\circ} \mathrm{C}$. The supernatants were obtained and used as the soluble fraction. An aliquot $(100 \mu 1)$ of the supernatant was loaded on the TSK GEL SW3000 column (Tosoh Corporation, Tokyo, Japan) and eluted with $50 \mathrm{mM}$ Bicine-ammonium ( $\mathrm{pH} \mathrm{8.4)} \mathrm{at} \mathrm{a} \mathrm{flow} \mathrm{rate} \mathrm{of} 0.8 \mathrm{ml} / \mathrm{min}$ (HP1100; Yokokawa Analytical Systems, Tokyo, Japan). The eluate was introduced directly into the ICP-MS instrument.

\section{Ethics of animal experiments}

This study was carried out in strict accordance with the recommendations in the guidelines for the care and use of laboratory animals set forth by our Institutional Animal Care and Use Committee (IACUC) of Japan National Institute of Occupational Safety and Health (JNIOSH). For mortality experiment, all mice were sacrificed 14 days after the injection using carbon dioxide. The protocol was approved by IACUC of JNIOSH (Permit Number: H22-02-2).

\section{Statistical analysis}

All data were presented as the mean \pm S.D. Statistical analyses were performed by ANOVA. Post-hoc TukeyKramer's multiple comparisons tests in a one-way ANOVA were employed to show which time points (ZT) were significantly different within each experimental group. $P$-values lower than 0.05 were considered evidence for statistical significance.

\section{RESULTS}

\section{Different severity of Cd-induced mortality and hepatotoxicity by injection time}

We reported earlier the diurnal variation of $\mathrm{Cd}$-induced mortality in our previous study in which ICR mice were sensitive during daytime while resistant during night time (Miura et al., 2012). In the present study, we first investigated the difference in $\mathrm{Cd}$-induced mortality by injection time using another strain, $\mathrm{C} 57 \mathrm{BL} / 6 \mathrm{~J}$ mice. Mice were received single injection of $\mathrm{CdCl}_{2}(6.4 \mathrm{mg} / \mathrm{kg}$; i.p. $)$ at 6 different time points (at ZT2, ZT6, ZT10, ZT14, ZT18, or ZT22) followed by monitoring the survival numbers until 14 days after the injection. As shown in Fig. 1-a, $\mathrm{CdCl}_{2}$ killed all the mice injected at ZT2. How- 
(a)

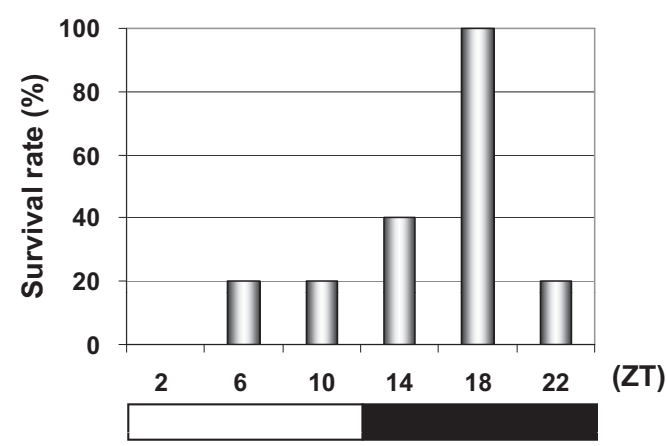

(b)

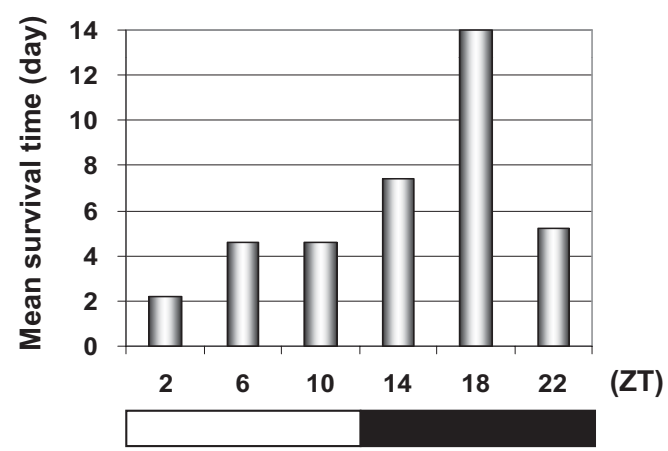

Fig. 1. Diurnal variation of Cd-induced mortality. Male C57BL/6J mice $(\mathrm{n}=5)$ were adapted for 14 days under the $12 \mathrm{~h}$ light cycle condition. Six groups of mice were received single i.p. injection of $\mathrm{CdCl}_{2}(6.4 \mathrm{mg} / \mathrm{kg})$ at each 6 different time points of a day (ZT2, ZT6, ZT10, ZT14, ZT18 or ZT22). (a) The survival rates until 14 days after the injection. (b) The mean survival time (MST, expressed as "day") estimated by KaplanMeier analysis.

ever, it was very surprisingly, all the mice survived at ZT18 injection. These survived mice were recovered their body weights to that of control levels until 14 days, and they seemed to be healthy, e.g. they moved actively and they had shiny fur as control group. The survival rate at ZT14 injection was also higher $(40 \%)$ than other injection time points except ZT18 injection. Furthermore, the mean survival time (MST; expressed as "day") estimated by Kaplan-Meier analysis was: 2.2 (ZT2); 4.6 (ZT6); 4.6 (ZT10); 7.4 (ZT14); 14.0 (ZT18) and 5.2 (ZT22) (Fig. 1-b). These results indicated that C57BL/6J mice showed extreme difference in mortality by injection timing, and were sensitive to $\mathrm{Cd}$-induced toxicity during light phase while tolerant during dark phase as well as our previous data using ICR mice (Miura et al., 2012). We did this mortality experiment only once from two reasons: 1) compliance of the $3 \mathrm{Rs}$ animal research principle;
2) acquisition of the same tendency (reproducibility) of diurnal variation compared to our previous data.

We next examined the difference in severity of hepatotoxicity by injection timing using lower dose of $\mathrm{Cd}$ (non-lethal dose: $4.5 \mathrm{mg} / \mathrm{kg}$ ). For the experimental convenience, we chose both ZT6 and ZT18 as injection time and used these times in the following experiments. Plasma samples were collected from $2 \mathrm{hr}$ to $24 \mathrm{hr}$ after the $\mathrm{Cd}$ injection at ZT6 or ZT18. As a result, at the ZT6 injection, the ALT values began to rise from $12 \mathrm{hr}$ and markedly increased at $24 \mathrm{hr}$ after the injection (Fig. 2, left). On the other hand, at ZT18 injection, the ALT values did not increased at all, not significantly different from the control level. This pattern was also observed by AST estimation (Fig. 2, right). Thus mice showed pronounced acute hepatotoxicity by $\mathrm{Cd}$ injection and had different severity to $\mathrm{Cd}$-induced hepatotoxicity by injection time. These results clearly indicate that the $\mathrm{Cd}$ sensitivity of mice is extremely different of a day.

\section{No differences in accumulation and distribution of $\mathbf{C d}$ in the liver}

To consider the mechanism of the diurnal variation of Cd-induced toxicity, we first investigated and compared the $\mathrm{Cd}$ accumulation level in the liver after the $\mathrm{Cd}$ injection, since liver is one of the target organs of acute $\mathrm{Cd}$ toxicity. Hepatic $\mathrm{Cd}$ levels were estimated using the liver samples collected at an appropriate time intervals after the $\mathrm{Cd}$ injection (same experiment as Fig. 2). As a result, the $\mathrm{Cd}$ levels which detected $2 \mathrm{hr}$ after the injection were not changed until $24 \mathrm{hr}$ after the Cd injection (Fig. 3). These accumulation patterns were not different by injection timing (ZT6 and ZT18). Therefore, Cd accumulation level in the liver and $\mathrm{Cd}$ uptake rate into the liver may not be the determination factors for the diurnal variation.

Next, we compared distribution profiles of hepatic $\mathrm{Cd}$ by the difference of injection time. The hepatic cytosol fractions of $\mathrm{Cd}$-injected mice were analyzed by HPLCICP/MS. The column (TSK gel G3000SW) used in this analysis can separate the two major MT isoforms (MT-1 and MT-2) in a single elution (Kobayashi et al., 2007). As shown in Fig. 4, Cd was detected mainly in the highmolecular-weight fraction (retention time 15 to $18 \mathrm{~min}$ ) at $2 \mathrm{hr}$ after the injection, then, came to be distributed almost in the MT-1 and MT-2 fractions (retention time approx. 22 and $25 \mathrm{~min}$ ) at $12 \mathrm{hr}$ after the injection. Similar distribution pattern was observed at $24 \mathrm{hr}$ after the injection (data not shown). These patterns were not different at all between two injection times (ZT6 and ZT18). Therefore, our results indicate that differences in the distribution of $\mathrm{Cd}$ in the liver are also not a determination factor for $\mathrm{Cd}$ - 
Cadmium chronotoxicity and glutathione
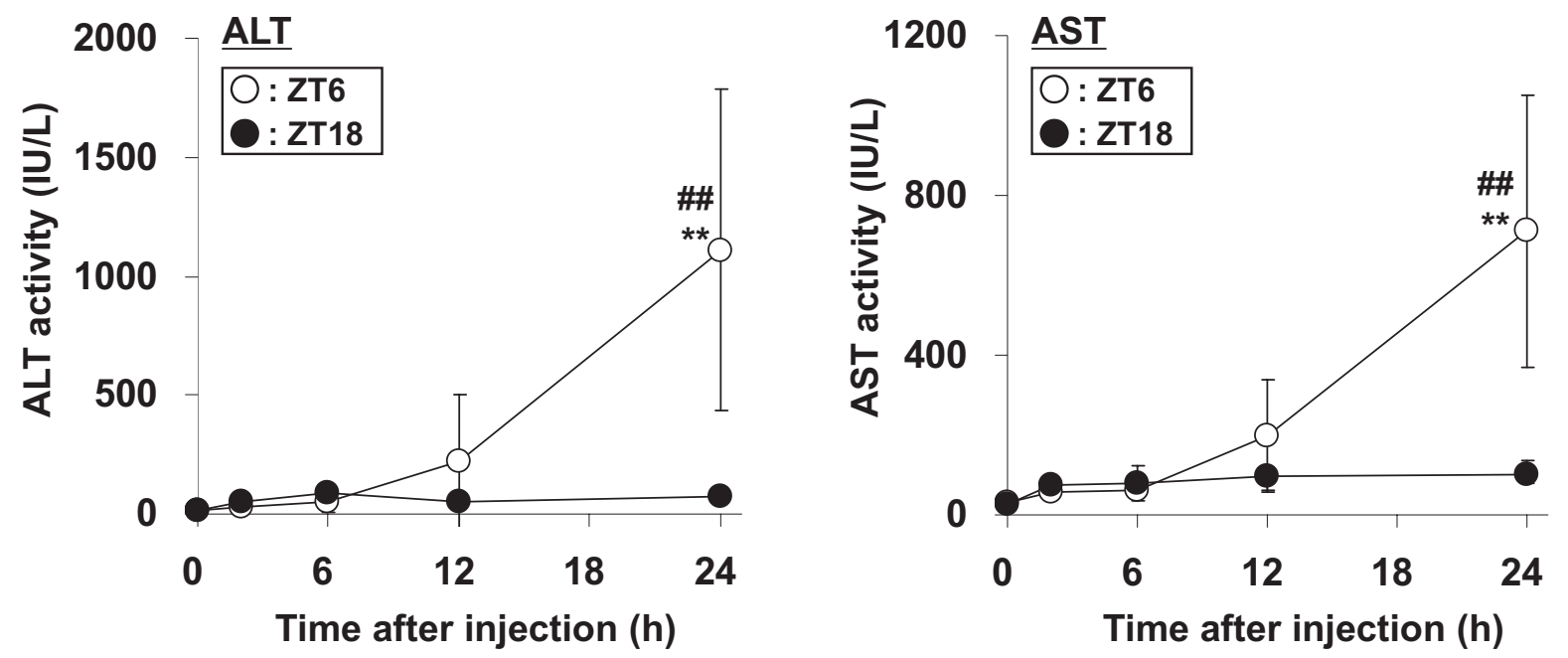

Fig. 2. Different severity of hepatotoxicity by injection times. Male C57BL/6J mice $(n=5)$ were received single i.p. injection of $\mathrm{CdCl}_{2}(4.5 \mathrm{mg} / \mathrm{kg})$ at ZT6 or ZT18. Control mice (for $\left.0 \mathrm{hr}\right)$ were received same volume $(0.1 \mathrm{ml} / 10 \mathrm{~g}$ body weight) of saline. Plasma samples were collected from $2 \mathrm{hr}$ to $24 \mathrm{hr}$ after the injection. The ALT and AST values were measured as indexes of hepatotoxicity. \#\# : vs. $0 \mathrm{hr}(\mathrm{p}<0.01),{ }^{* *}$ : vs. ZT18 $(\mathrm{p}<0.01)$

induced chronotoxicity.

\section{Non-contribution of MT for diurnal variation of Cd-induced toxicity}

The diurnal variations of levels of biological defense factors are thought to be taken part in determination of the Cd-induced chronotoxicity. Because MT is one of the most important defense factors against $\mathrm{Cd}$ toxicity, we first estimated the diurnal variation of MT protein lev$\mathrm{el}$ in the liver of non-treated mice. As a result, although the diurnal variation of hepatic levels of MT protein was observed, this variation was not correlated to the diurnal variation of $\mathrm{Cd}$ sensitivity (Fig. 5-a). Therefore, the basal (non-induced) level of hepatic MT is hard to think as a determination factor for Cd-induced chronotoxicity. Moreover, since MT is an inducible protein (Ashino et al., 2003; Haq et al., 2003) and its induction level after the injection is known to be important, we compared the MT induction pattern by the difference of injection times of Cd. As shown in Fig. 5-b, MT protein levels rose up 6 $\mathrm{hr}$ after the $\mathrm{Cd}$ injection, and kept increasing until $24 \mathrm{hr}$. This induction pattern was completely fit closely between both injection times (ZT6 and ZT18). From these results, in addition to MT basal level, there were no differences in the MT induction level and induction speed between both injection times. Therefore we can exclude the possibility of MT contribution for Cd-induced chronotoxicity.

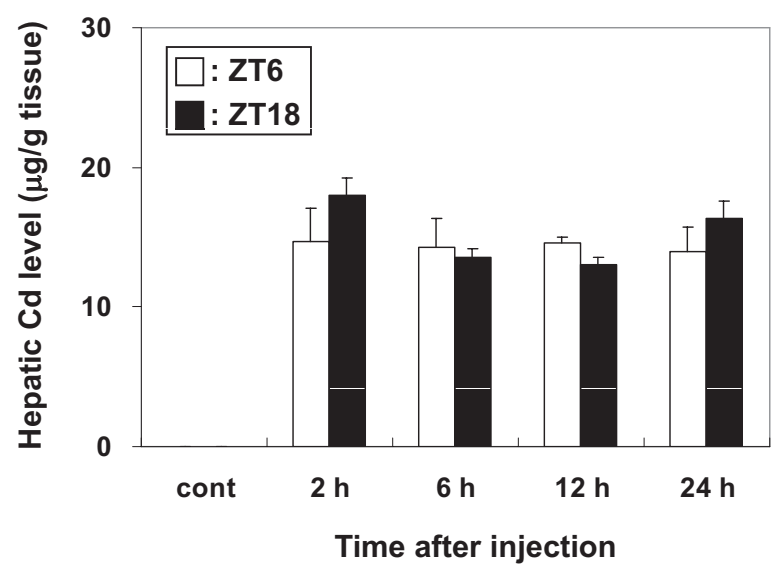

Fig. 3. Accumulation and uptake rate of $\mathrm{Cd}$ into the liver after the Cd injection. Hepatic Cd levels were measured by ICP-MS using the liver samples obtained from the time-course experiment in Fig. 2. Hepatic Cd levels were estimated by ICP-MS as described in Material and methods.

\section{Contribution of GSH for diurnal variation of Cd-induced toxicity}

We further examined the contribution of GSH, another biological defense factor for Cd toxicity. Using same samples from Fig. 5-a, the hepatic basal levels of GSH 

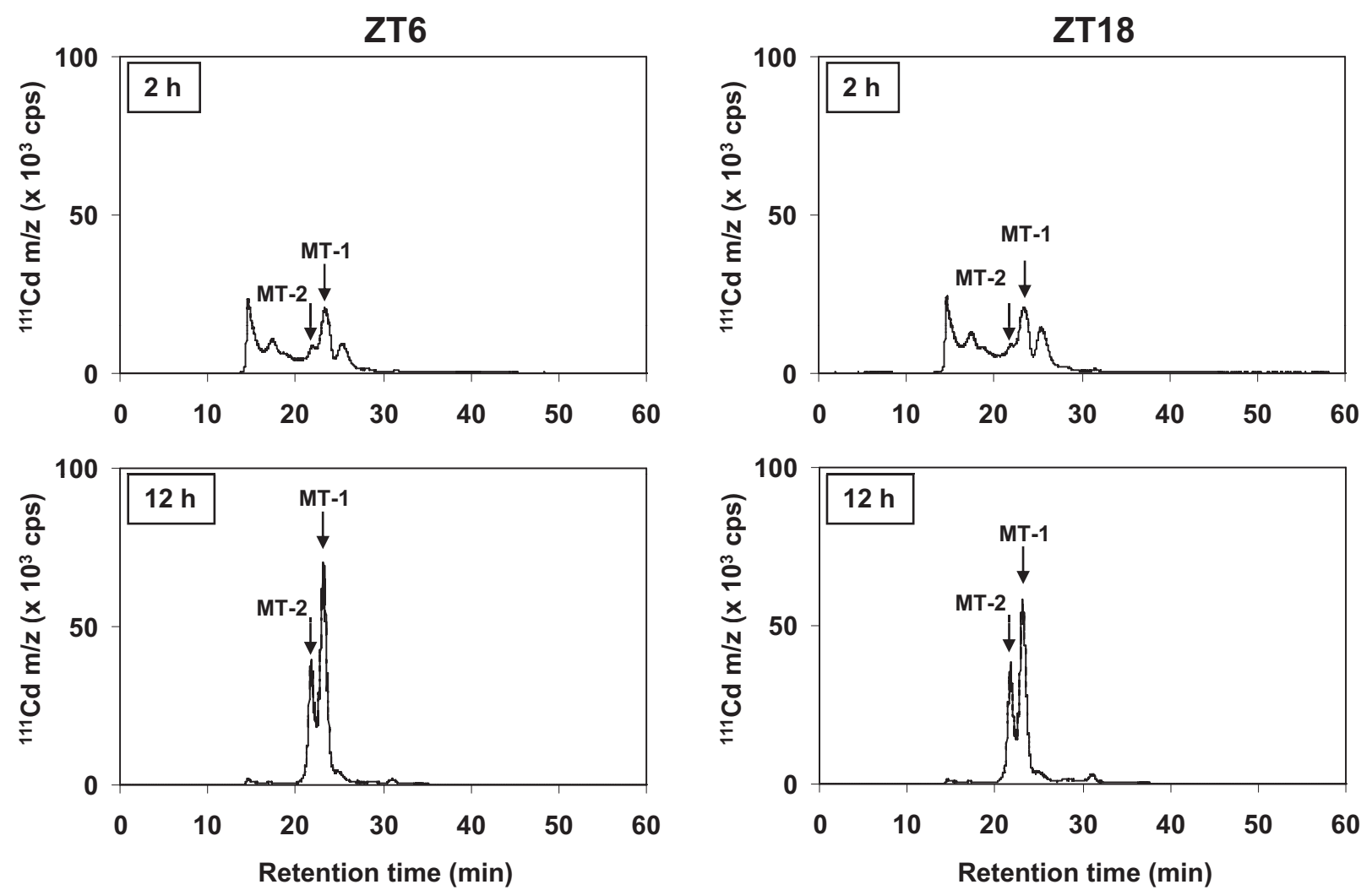

Fig. 4. Distribution profiles of $\mathrm{Cd}$ in the liver cytosol after the $\mathrm{Cd}$ injection. Using the liver samples obtained from the time-course experiment in Fig. 2, cytosol samples were prepared after ultracentrifugation (105,000 x g for $1 \mathrm{hr}$ ) of liver homogenates. The cytosol samples were loaded on a TSK GEL SW3000 column and eluted. The eluates were introduced directly into the ICP-MS instrument. The distribution profiles of Cd were determined at mass numbers of $111 \mathrm{~m} / \mathrm{z}$, and indicated as count per second (cps). Positions of the two major MT isoforms (MT-1 and MT-2) were indicated by arrows.

were estimated. As a result, although hepatic GSH levels showed significant diurnal variation, this variation was not correlated to the diurnal variation of $\mathrm{Cd}$ sensitivity (Fig. 6-a). Therefore, as the result of MT (Fig. 5-a), we think that the basal level of hepatic GSH is not a determination factor for Cd-induced chronotoxicity. However, we observed that the fluctuation levels of GSH after $\mathrm{Cd}$ injection had a difference between injection times. In ZT6 injection group, the level of GSH decreased about half of control level $6 \mathrm{hr}$ after the injection (significantly different from ZT18 group) and subsequent its recovery seemed to be low or delayed (Fig. 6-b). On the other hand, no decrease was observed in ZT18 injection group. This result suggested the contribution of GSH.

We subsequently examined the GSH contribution by additional estimation. To prove this, we tried to deplete the hepatic GSH level using buthionine sulfoximine
(BSO), a specific inhibitor of glutamate-cysteine ligase (Griffith et al., 1979). Male C57BL/6J mice were administered $20 \mathrm{mM}$ of BSO in drinking water for four days to deplete the GSH continuously (Watanabe et al., 2003). By this administration, the hepatic GSH level decreased about half of the control level (Fig. 7-b, left). BSO gave no effect on body weight (data not shown). Under this condition, mice were received single $\mathrm{Cd}$ injection ( $4.5 \mathrm{mg} / \mathrm{kg}$; i.p.) at ZT6 or ZT18 followed by collection of plasma $24 \mathrm{hr}$ after the injection and estimation of ALT activities. Body weights, liver weights and both values of ALT and ALT were not significantly changed by BSO treatment alone (data not shown). As a result, the ALT value increased at ZT6 while was lower at ZT18 in the BSO-non-administered group, as well as former (Fig. 7-b, middle). However, this difference was disappeared in the BSO-administered group (Fig. 7-b, right). BSO also abol- 
Cadmium chronotoxicity and glutathione

(a)

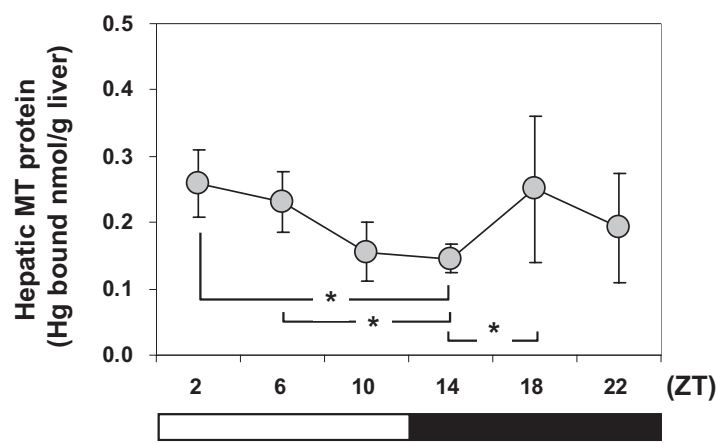

(b)

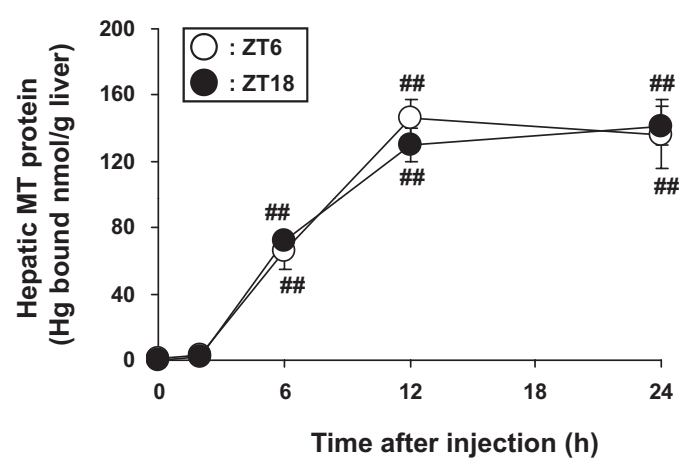

Fig. 5. Basal and induction levels of hepatic MT. (a) Diurnal variation of basal MT protein level. Male C57BL/6 J mice which had not been received any administrations were dissected at 6 different ZT $(2,6,10,14,18$ or 22). Liver samples were collected followed by determining the MT protein levels. Data was represented as mean \pm S.D. *, significantly different from compared values ( $p<0.05$ ). (b) Comparison of MT induction levels after Cd injection at ZT6 or ZT18. Hepatic MT levels were measured using the liver samples obtained from the timecourse experiment in Fig. 2. \#\# : vs. 0 hr $(\mathrm{p}<0.01)$.

ished the diurnal variation of hepatotoxicity estimated by AST (data not shown). From these results, the hepatic GSH level may be one of the candidate factors for determination of the Cd-induced diurnal variation.

\section{DISCUSSION}

Our results clearly demonstrated that mice had extreme diurnal variation of $\mathrm{Cd}$-induced hepatotoxicity in addition to mortality (Fig. 1 and Fig. 2); mice seemed to be sensitive to Cd-induced toxicity during light phase while tolerant during dark phase. To consider the mechanisms of this (a)

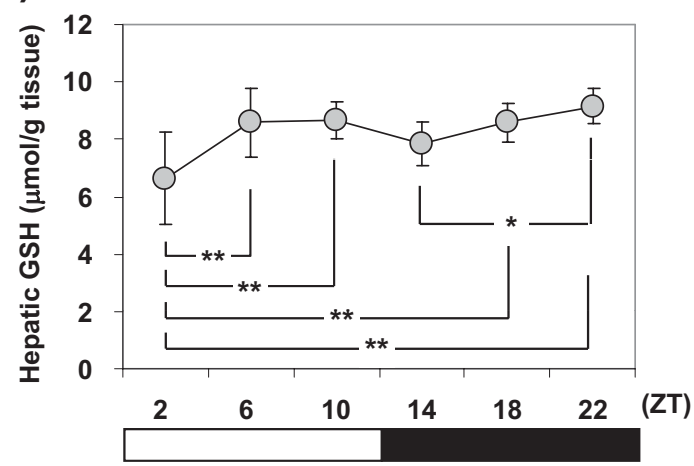

(b)

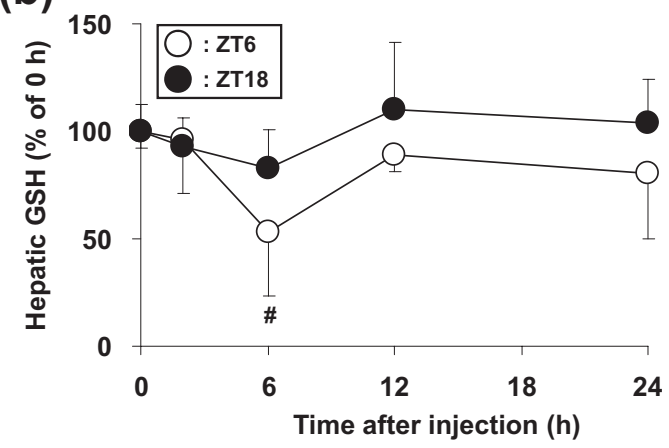

Fig. 6. Basal levels and fluctuation levels of hepatic GSH. (a) Diurnal variation of basal GSH level. Hepatic GSH levels were measured using the liver samples obtained from the non-treated mice in Fig. 5-a. Data was represented as mean \pm S.D. $*, * *$, significantly different from compared values $(*: \mathrm{p}<0.05 ; * *$ : $\mathrm{p}<0.01)$. (b) Changes in hepatic GSH levels after Cd injection at ZT6 or ZT18. Hepatic GSH levels were measured using the liver samples obtained from the time-course experiment in Fig. 2. \# : vs. $0 \mathrm{hr}(\mathrm{p}<0.05)$.

chronotoxicity, we first examined the Cd levels in the liver, since acute $\mathrm{Cd}$ exposure shows hepatotoxicity in mammals (Waalkes et al., 1992; Hiratsuka et al., 1993; Honda et al., 2010). As a result, both injection times (ZT6 and ZT18) showed same pattern of fluctuation levels of $\mathrm{Cd}$ in the liver (Fig. 3) and distributions of Cd in hepatic soluble fractions (Fig. 4). Therefore, we think that differences of the $\mathrm{Cd}$ accumulation in the liver, the $\mathrm{Cd}$ distribution in the liver cytosol, the $\mathrm{Cd}$ uptake into the liver, and the $\mathrm{Cd}$ distribution in the hepatic cytosol are not attributed to this diurnal variation.

It is well known that biological defense factors show 
(a)

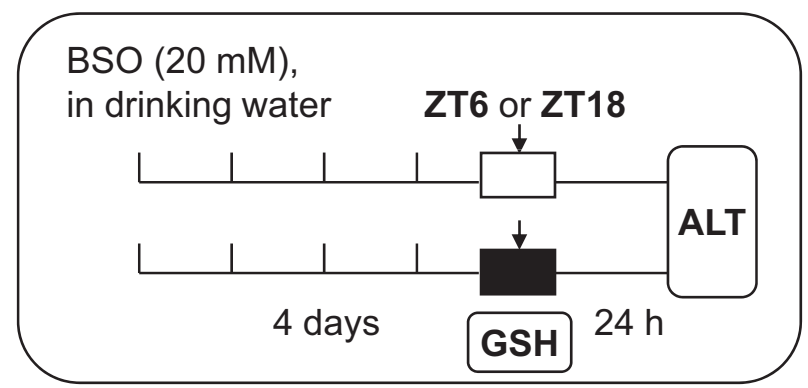

(b)
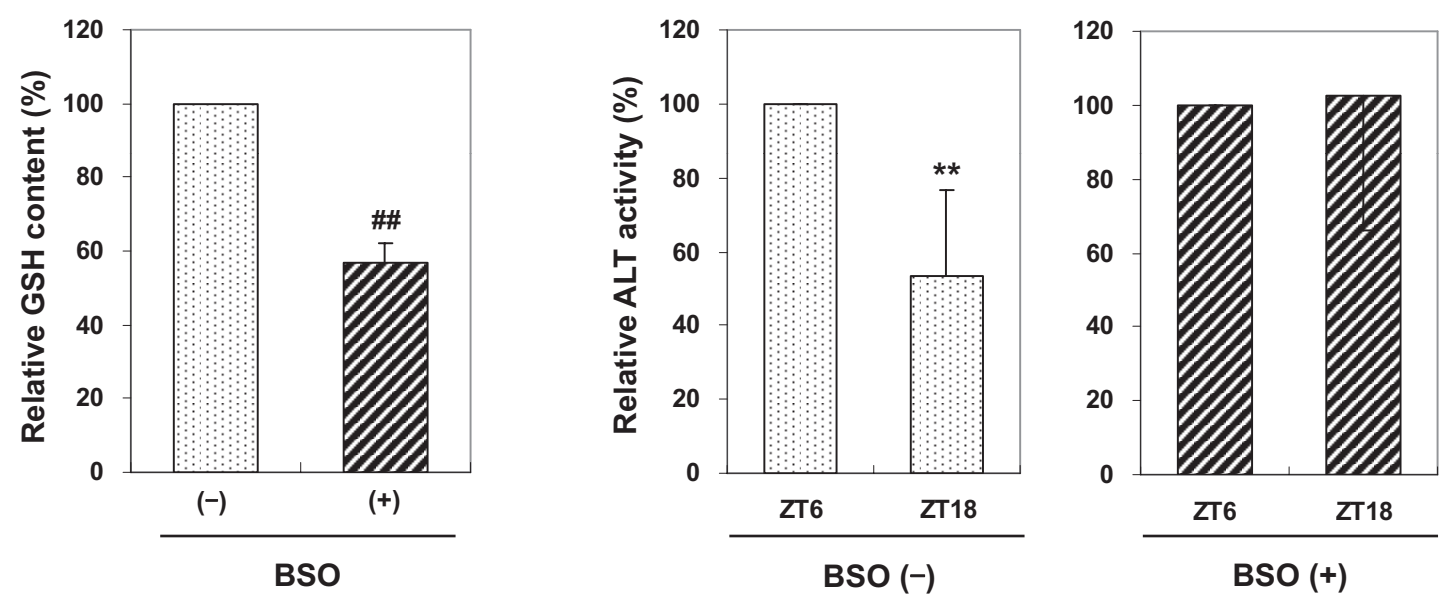

Fig. 7. Examination of GSH contribution on diurnal variation of Cd-induced toxicity. (a) Schematic experimental design of BSO administration and Cd injection. (b) Effect of GSH depletion on Cd-induced diurnal sensitivity. Male C57BL/6J mice were given free access to drinking water supplemented by $20 \mathrm{mM}$ BSO for 4 days to prevent GSH synthesis continuously. Liver samples were collected followed by determining the GSH levels. Then, mice were injected with $\mathrm{CdCl}_{2}(4.5 \mathrm{mg} / \mathrm{kg}$ i.p.). Plasma samples were collected $24 \mathrm{hr}$ after the injection followed by measuring the ALT activity. \#\#: vs. BSO $(-)(\mathrm{p}<0.05)$, **: vs. ZT6 $(\mathrm{p}<0.01)$.

diurnal variations in their expression levels or their activities. For example, expressions of many drug processing genes (Zhang et al., 2009) and antioxidant genes including MT and GSH (Xu et al., 2012) show diurnal variation in the mouse liver. MT is a most important defensive protein with high affinity for heavy metals such as $\mathrm{Cd}$ via abundant cysteine residues in its molecule (Palmiter, 1998; Fujiwara et al., 2011), and is induced by Cd exposure at transcriptional level, thus takes part in detoxification of Cd (Haq et al., 2003; Nakamura et al., 2012). Our present work showed that the diurnal variation of hepatic MT level in non-treated mice was not correlated to the diurnal variation of Cd sensitivity (Fig. 5-a), and MT induction patterns (induction level and induction speed) were also not different between both injection times (ZT6 and ZT18; Fig. 5-b). Therefore, we came to the conclusion that MT had no contribution for determination of $\mathrm{Cd}$-induced chronotoxicity. On the other hand, the hepatic GSH level after Cd injection was significantly lower at ZT6 injection than the level at ZT18 injection (Fig. 6-b), although we could not find relationship between the diurnal variation of hepatic GSH level and the Cd susceptibility (Fig. 6-a). The subsequent GSH-depletion experiment using BSO (Fig. 7) suggested the contribution of GSH to the Cd-induced chronotoxicity. Therefore, we think that the hepatic GSH was a candidate factor for determining the $\mathrm{Cd}$-induced chronotoxicity. There are several reports which describe the diurnal variation of 
Cadmium chronotoxicity and glutathione

expression levels of enzymes involved in GSH biosynthesis, such as $\gamma$-glutamylcysteine synthetase (for synthesis) and $\gamma$-glutamyl transferase (for degradation) (White et al., 1987); also, GSH-related enzymes like glutathione S-transferase and glutathione peroxidase (Xu et al., 2012). These proteins may contribute to $\mathrm{Cd}$-induced toxicity by concerning with GSH complexly, therefore, we are going to examine the $\mathrm{Cd}$-induced fluctuation of these protein levels and consider the contribution to the diurnal variation of $\mathrm{Cd}$ toxicity. There are many reports indicating the diurnal variation of GSH levels in plasma in addition to liver (Jaeschke and Wendel, 1985; Blanco et al., 2007; Xu et al., 2012). We estimated the hepatic levels of GSH in this report, therefore, we need further information about the GSH levels in plasma. Moreover, because Cdinduced chronotoxicity may not be explain only by the rhythms of the detoxification systems, we should consider and pay attention to $\mathrm{Cd}$-induced effects on other rhythmic factors starting with metabolic systems in addition to whole body biological rhythms (Caride et al., 2010; Jimenez-Ortega et al., 2011; Lafuente, 2013).

At the present stage, we presume that the priority may be given to clarify the existence of diurnal variation of susceptibility to metal compounds by many researchers, since there are a few reports examined the Cd-induced chronotoxicity: using mice (in French) (Cambar et al., 1983) or Paramecium tetraurelia (Hinrichsen and Tran, 2010) in addition to our reports (Miura, 2009; Ohtani et al., 2013). The information acquired from these examinations is important for protecting the human health from the hygienic viewpoint. In modern society, the "shift work with night work" is adopted not only into manufacturing industries but into various fields, and has been an indispensable working arrangement. Actually, there are $27.3 \%$ of shift workers in Japan, that is, about one quarter or one third of the all workers (in 2007, Survey on State of Employees' Health, Ministry of Health, Labour and Welfare). It is noteworthy that, in steel industry where workers have many opportunities to expose to metal compounds, the percentage of companies which adopt the "shift work with night work" is reaching to seven out of ten $(64.3 \%$ in 2005 , General Survey on Working Conditions, Ministry of Health, Labour and Welfare). Shift workers work at day time and night time, therefore, they have possibilities to be exposed to toxic substances at various times of a day. Our present research propounds a new agenda, which is unnecessary to consider under the normal conditions (environmental levels are fewer than regulation values) but is necessary in the case of accidental exposure such as explosion or spillage in which workers are exposed to high concentration (tox- ic dose) of toxic substances. Shift workers will need to pay more attention to exposable substances during higher sensitive time, because our data indicated extreme differences in diurnal variation: all-or-none of mortality and hepatotoxicity. By introducing our "chronotoxicological" viewpoint, our data could apply in higher-precision of risk management to shiftworkers. Moreover, if the diurnal variation of sensitivity is exist among many chemical compounds, the regulation values such as "No Observed Adverse Effect Level" (NOAEL) may be reconsidered because the current regulation values usually use the results of experiments conducted during daytime.

At this time, however, it is difficult to extrapolate the experimental data obtained from mice to human beings. In the case of the medical field involving chronopharmacology and chronotherapy (Burioka et al., 2010; Flack and Nasser, 2011), it may be easier than the case of toxicological field to extrapolate experimental data to human beings because the experimental object substances are medicinal drugs and can be applied directly to human beings. In the toxicological field, however, information regarding to biological effects of toxic substances to human body must be acquired from animal experiments since toxic substances are never given directly to human beings for anything. Recently, chronotoxicological cohort study was reported which described that the timing of poisoning ingestion (in this case, oleander poisoning) affected the survival in humans (Carroll et al., 2012). Therefore, it is important to determine the mechanism of this $\mathrm{Cd}$-induced chronotoxicity. For example, if the diurnal variation is only the difference in action rhythms such as feeding diet between mice and human, we may simply understand that human beings are more sensitive to Cd-induced toxicity during night, since mice are nocturnal animal but human beings are diurnal. If different mechanism(s) is concerned, we cannot simply extrapolate the reversing of action rhythm as mentioned above. Moreover, considering of exposure to workers in the work places, we think that examinations not only by i.p injection (in this study) but by other administration routes are required. At the present, we have obtained one result which shows $\mathrm{Cd}$-induced chronotoxicity by subcutaneous injection (in preparation).

In conclusion, we presented here that the $\mathrm{Cd}$-induced toxicity such as hepatotoxicity and mortality showed extremely different by the injection times of a day. This diurnal variation was not by differences in hepatic Cd level, hepatic MT level, and MT induction level along with induction speed. One of the determination factors which we suggested is GSH. We think that this extreme diurnal variation, all-or-none of mortality and hepatotoxicity, are not explained by GSH alone, therefore we are advancing 
the elucidation of the mechanism by examination of the contribution of other factors especially for antioxidants. Our modern chronotoxicological viewpoint could contribute with new concepts to classic toxicology.

\section{ACKNOWLEDGMENTS}

We thank Dr. Masaya Takahashi and Dr. Yasutaka Ogawa, Japan National Institute of Occupational Safety and Health (JNIOSH), Kawasaki, Japan for helpful advice to and coordinating of our project team. This work was supported by grant-in-aid for project research (P21-01) and fundamental research (F24-01) from JNIOSH.

\section{REFERENCES}

Ashino, T., Ozawa, S., Numazawa, S. and Yoshida, T. (2003): Tissue-dependent induction of heme oxygenase-1 and metallothionein-1/2 by methyl methanesulfonate. J. Toxicol. Sci., 28, 181-189.

Blanco, R.A., Ziegler, T.R., Carlson, B.A., Cheng, P.Y., Park, Y., Cotsonis, G.A., Accardi, C.J. and Jones, D.P. (2007): Diurnal variation in glutathione and cysteine redox states in human plasma. Am. J. Clin. Nutr., 86, 1016-1023.

Burioka, N., Fukuoka, Y., Koyanagi, S., Miyata, M., Takata, M., Chikumi, H., Takane, H., Watanabe, M., Endo, M., Sako, T., Suyama, H., Ohdo, S. and Shimizu, E. (2010): Asthma: Chronopharmacotherapy and the molecular clock. Adv. Drug Deliv. Rev., 62, 946-955.

Cambar, J., Cal, J.C., Desmouliere, A. and Guillemain, J. (1983): Circadian variations of the mortality of mice due to cadmium sulfate. C R Seances. Acad. Sci. III, 296, 949-952.

Caride, A., Fernandez-Perez, B., Cabaleiro, T., Tarasco, M., Esquifino, A.I. and Lafuente, A. (2010): Cadmium chronotoxicity at pituitary level: effects on plasma ACTH, GH, and TSH daily pattern. J. Physiol. Biochem., 66, 213-220.

Carroll, R., Metcalfe, C., Gunnell, D., Mohamed, F. and Eddleston, M. (2012): Diurnal variation in probability of death following self-poisoning in Sri Lanka--evidence for chronotoxicity in humans. Int. J. Epidemiol., 41, 1821-1828.

Flack, J.M. and Nasser, S.A. (2011): Benefits of once-daily therapies in the treatment of hypertension. Vasc, 7, 777-787.

Froy, O. (2009): Cytochrome P450 and the biological clock in mammals. Curr. Drug Metab., 10, 104-115.

Fujiwara, Y., Banno, H., Shinkai, Y., Yamamoto, C., Kaji, T. and Satoh, M. (2011): Protective effect of pretreatment with cilostazol on cytotoxicity of cadmium and arsenite in cultured vascular endothelial cells. J. Toxicol. Sci., 36, 155-161.

Griffith, O.W., Anderson, M.E. and Meister, A. (1979): Inhibition of glutathione biosynthesis by prothionine sulfoximine (S-n-propyl homocysteine sulfoximine), a selective inhibitor of gammaglutamylcysteine synthetase. J. Biol. Chem., 254, 1205-1210.

Haq, F., Mahoney, M. and Koropatnick, J. (2003): Signaling events for metallothionein induction. Mutat. Res., 533, 211-226.

Hermida, R.C., Ayala, D.E. and Calvo, C. (2005): Administration-time-dependent effects of antihypertensive treatment on the circadian pattern of blood pressure. Curr. Opin. Nephrol.
Hypertens., 14, 453-459.

Hinrichsen, R.D. and Tran, J.R. (2010):A circadian clock regulates sensitivity to cadmium in Paramecium tetraurelia. Cell. Biol. Toxicol., 26, 379-389.

Hiratsuka, H., Katsuta, O., Iwata, H., Matsumoto, J. and Umemura, T. (1993): Acute toxicity of cadmium in rats with or without cadmium pretreatment. J. Toxicol. Sci., 18, 197-201.

Honda, A., Komuro, H., Hasegawa, T., Seko, Y., Shimada, A., Nagase, H., Hozumi, I., Inuzuka, T., Hara, H., Fujiwara, Y. and Satoh, M. (2010): Resistance of metallothionein-III null mice to cadmium-induced acute hepatotoxicity. J. Toxicol. Sci., 35, 209215.

Jaeschke, H. and Wendel, A. (1985): Diurnal fluctuation and pharmacological alteration of mouse organ glutathione content. Biochem. Pharmacol., 34, 1029-1033.

Jimenez-Ortega, V., Cano-Barquilla, P., Scacchi, P.A., Cardinali, D.P. and Esquifino, A.I. (2011): Cadmium-Induced Disruption in 24-h Expression of Clock and Redox Enzyme Genes in Rat Medial Basal Hypothalamus: Prevention by Melatonin. Front Neurol., 2, 1-9.

Kobayashi, K., Kuroda, J., Shibata, N., Hasegawa, T., Seko, Y., Satoh, M., Tohyama, C., Takano, H., Imura, N., Sakabe, K., Fujishiro, H. and Himeno, S. (2007): Induction of metallothionein by manganese is completely dependent on interleukin-6 production. J. Pharmacol. Exp. Ther., 320, 721-727.

Koyanagi, S., Nakagawa, H., Kuramoto, Y., Ohdo, S., Soeda, S. and Shimeno, H. (2003): Optimizing the dosing schedule of TNP470 [O-(chloroacetyl-carbamoyl) fumagillol] enhances its antitumor and antiangiogenic efficacies. J. Pharmacol. Exp. Ther., 304, 669-674.

Lafuente, A. (2013): The hypothalamic-pituitary-gonadal axis is target of cadmium toxicity. An update of recent studies and potential therapeutic approaches. Food Chem. Toxicol., 59, 395-404.

Miura, N., Kanayama, Y., Nagai, W., Hasegawa, T., Seko, Y., Kaji, T. and Naganuma, A. (2006): Characterization of an immortalized hepatic stellate cell line established from metallothioneinnull mice. J. Toxicol. Sci., 31, 391-398.

Miura, N. (2009): Individual susceptibility to cadmium toxicity and metallothionein gene polymorphisms: with references to current status of occupational cadmium exposure. Ind. Health, 47, 487494.

Miura, N., Yanagiba, Y., Ohtani, K., Mita, M., Togawa, M. and Hasegawa, T. (2012): Diurnal variation of cadmium-induced mortality in mice. J. Toxicol. Sci., 37, 191-196.

Nakamura, Y., Ohba, K., Suzuki, K. and Ohta, H. (2012): Health effects of low-level cadmium intake and the role of metallothionein on cadmium transport from mother rats to fetus. J. Toxicol. Sci., 37, 149-156.

Ohtani, K., Yanagiba, Y., Ashimori, A., Takeuchi, A., Takada, N., Togawa, M., Hasegawa, T., Ikeda, M. and Miura, N. (2013): Influence of injection timing on severity of cadmium-induced testicular toxicity in mice. J. Toxicol. Sci., 38, 145-150.

Palmiter, R.D. (1998): The elusive function of metallothioneins. Proc. Natl. Acad. Sci. USA, 95, 8428-8430.

Peters, G.J., Van Dijk, J., Nadal, J.C., Van Groeningen, C.J., Lankelma, J. and Pinedo, H.M. (1987): Diurnal variation in the therapeutic efficacy of 5-fluorouracil against murine colon cancer. In Vivo, 1, 113-117.

Pinot, F., Kreps, S.E., Bachelet, M., Hainaut, P., Bakonyi, M. and Polla, B.S. (2000): Cadmium in the environment: sources, mechanisms of biotoxicity, and biomarkers. Rev. Environ. Health, 15, 
Cadmium chronotoxicity and glutathione

299-323.

Waalkes, M.P., Coogan, T.P. and Barter, R.A. (1992): Toxicological principles of metal carcinogenesis with special emphasis on cadmium. Crit. Rev. Toxicol., 22, 175-201.

Wade, M.J., Davis, B.K., Carlisle, J.S., Klein, A.K. and Valoppi, L.M. (1993): Environmental transformation of toxic metals. Occup. Med., 8, 574-601.

Watanabe, T., Sagisaka, H., Arakawa, S., Shibaya, Y., Watanabe, M., Igarashi, I., Tanaka, K., Totsuka, S., Takasaki, W. and Manabe, S. (2003): A novel model of continuous depletion of glutathione in mice treated with L-buthionine $(\mathrm{S}, \mathrm{R})$-sulfoximine.
J. Toxicol. Sci., 28, 455-469.

White, B.P., Davies, M.H. and Schnell, R.C. (1987): Circadian variations in hepatic glutathione content, gamma-glutamylcysteine synthetase and gamma-glutamyl transferase activities in mice. Toxicol. Lett., 35, 217-223.

Xu, Y.Q., Zhang, D., Jin, T., Cai, D.J., Wu, Q., Lu, Y., Liu, J. and Klaassen, C.D. (2012): Diurnal variation of hepatic antioxidant gene expression in mice. PLoS One, 7, e44237.

Zhang, Y.K., Yeager, R.L. and Klaassen, C.D. (2009): Circadian expression profiles of drug-processing genes and transcription factors in mouse liver. Drug Metab. Dispos., 37, 106-115. 